\title{
Desenvolvimento distribuído de software para sistemas pervasivos: um estudo de caso
}

Tre L'Erario ${ }^{1,2}$, Tiago Faustino Prado ${ }^{1}$, Mauro de Mesquita Spinola ${ }^{1}$, Marcelo Schneck de Paula Pessôa ${ }^{1}$, José Augusto Fabri ${ }^{1,2}$

Engenharia de Produção - Escola Politécnica da Universidade de São Paulo Av. Professor Almeida Prado, trav 2 n. 128 CEP 05508-900

Cidade Universitária São Paulo - Brasil Tel. 5511 3091-5363

Centro de Pesquisas em Informática da Fundação Educacional do Município de Assis. Av. Getúlio Vargas, 1200 Vila Nova Santana

Assis - SP - Brasil CEP: 19807-634 - Fone/Fax: (18) 3302-1055

lerarioffemanet.com.br, tiagopoli@uol.com.br, mauro.spinolaepoli.usp.br mpessoa@usp.br, fabri@femanet.com.br

Resumo. Este artigo aborda o desenvolvimento distribuido de componentes para aplicações pervasivas. É ilustrado de que modo os componentes de software podem agregar funcionalidades a dispositivos móveis, tais como celulares, handhelds, e outros, além do impacto que estes tipos de agregacões têm no processo de desenvolvimento distribuido. Este trabalho apresenta um estudo de caso onde um processo distribuido foi utilizado para construir uma aplicação.

Palavras Chaves: Componentes Pervasivos, Produção Distribuida, Processo Distribuido de Software.

Abstract. This article is related to distributed development of components for pervasive applications. It shows how the software components can add functionalities to mobile devices (such as cell phones, handhelds, and a few others), as well as the impact that these types of aggregations have on the distributed development process. This work presents a case study in which a distributed process was used to build up an application.

Keywords: Pervasive components, Distributed Production, Distributed Software Process.

\section{Introdução}

A Tecnologia de Informação móvel é uma ferramenta capaz de permitir a distribuição ordenada da informação provendo auxílio a negócios, comodidade e agilidade a transações, que exigem versatilidade e velocidade. Atualmente, o termo "pervasivo" é empregado para abordar a façanha de distribuição, de mobilidade e de transparência da computação [Burkhardt 2002][Hansmann 2001]. Neste cenário, o usuário tem acesso à informação em qualquer dispositivo que não necessariamente seja um computador desktop convencional.

No entanto, o desenvolvimento de aplicações pervasivas é direcionado por um conjunto de requisitos não funcionais. Tais requisitos são resultantes das limitacõos capacidades dos equipamentos que, ainda em sua maioria, apresentam características 
heterogêneas. Agregar funcionalidades a estes dispositivos significa instalar componertes fisicas. Por esta razão, as informaç̃es ocal e/ou distribuído adequado a suas caractentes sace acessadas em um computador cacteristic in inclui, por exemplo, a redução de casas decimais e o ajuste da resolca âteis. Este tratamo outros.

Politécnica da USP, em parceria Departamento de Engenharia de Produção da atualmente pesquisa o desenvolvimento e o processo de como um de seus projetos pioneiros, serviu de sub deftware. O projeto ManWA tecnologias. Entre estes processos destaca-s subsidio para aplicação de process mplementacão de umaplicativocentimento distribuído e mo possível

Este artigo descreve um estudo de caso do desivana elaborado de maneira distribuída, em que vários nós deimento de um sistema pervasivo, independente de software) agregarm de rede (unidades de produrĩ desenvolvimento foi de pda's e celulares, desconsiderando do projeto, desenvolvidas para eletrodomésticos e demais dispositivos que nãorderando aplicações categorias. É abordado o processo de produção disposivos que não se enquadram nestas dispositivos móveis/portáteis.

\subsection{Arquitetura do software utilizado no estudo de caso}

A arquitetura multicamada foi adotada no projeto ManWApp com o objetiv capacidade de escalonamento, distribuicão princípio, foram concebidos somente alguns requisitos, a posteriormente, foram adaptados concebidose, foram adaptados para dispositivos portáteis. Outros requisitos foram concebidos inicialmente para ambos os equipamentos. Alguns requisitos foram projetados impactos no projeto (exemplo: para implementar nó). A Figura 1 a seguir ilustro subproduto um nó pode precisar de um subproduto de outro Camadas utilizada no ManWApp.

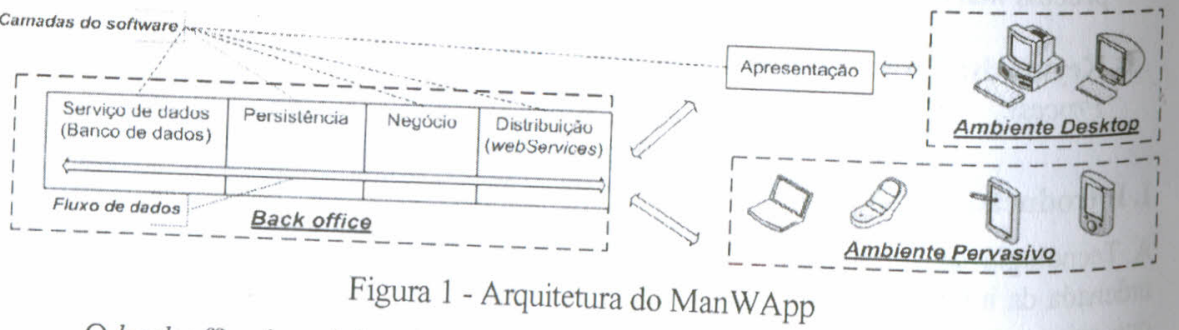

ManWApp. O ambiente desto conjunto de funcionalidades (como regras de negócio) do envolve qualquer computador convencional, que tenh acesso a aplicação por meio de um navegador web e que, por isso, dispensa a instalação de módulos de software. O ambiente pervasivo é composto pelo conjunto de dispositivos móveis/portáteis que acessam a aplicação.
A camada de apresentação gera a interface visível do programa, que é utilizada pelo A carnar a aplicação. As regras de negócio determinam de que maneira os dado usuário para aces. Na camada de persistência tem-se o acesso ao serviço de dados, no qual serão utiliza informação necessária para o funcionamento da aplicação. A camada de reside toda a in distribulção ( através de proto Com o uso de web services, é possivel acessar dados em diversas aplicações, de tecnologis com tecnologias diferentes e apresentar o resultado final, na forma de aplicação desenvolvida um usuário específico [Newcomer 2002].

Nos dispositivos móveis/portáteis, foi necessário desenvolver e instalar um módulo de oftware. Este software contém componentes que prevêem as limitações do dispositivo tai como o tamanho da tela, a limitação de processamento, de memória, de comunicação, etc.

\section{Sistemas pervasivos}

A finalidade da computação pervasiva é aproximar o sistema do usuário de maneira que haja interações imediatas. Seu princípio básico de arquitetura funcional está alicerçado no mesmo princípio dos sistemas distribuídos. Este modelo computacional utiliza-se da descentralização para obter melhor performance e oferecer uma gama maior de ferramentas, que auxiliam as pessoas a resolverem problemas de maneira rápida e aparentemente simples, do ponto de vista do usuário [Hansmann 2001].

Hansmann [Hansmann 2001] e Burkhardt [Burkhardt 2002] definem que os sistemas pervasivos são constituídos da descentralização, diversificação, conectividade e simplicidade. A descentralização é uma característica na qual as informações e/ou o processamento podem ser distribuídos. Os usuários de um sistema pervasivo podem obter informações a partir de dispositivos diferentes, tais como pagers, telefone celular, pda's entre outros dispositivos. Todo dispositivo, por mais diferente que seja, precisa estar conectado logicamente, pois é necessário buscar informações remotas ou enviar dados armazenados. Além disso, os aplicativos precisam ser simples, pois o usuário precisa ter acesso fácil a informações. Estes itens são considerados não só no desenvolvimento de componentes para dispositivos móveis/portáteis, mas também no desenvolvimento do sistema back office pois, ambos trocam dados que são, de alguma maneira, disponibilizados para o usuário por meio do dispositivo portátil.

As limitações dos dispositivos são requisitos não funcionais, que precisam set consideradas no projeto. Utilizando estes equipamentos, o usuário tem o sistema em qualquer lugar e hora dependendo é claro da disponibilidade de comunicação e do servidor. No entanto, quase que a totalidade dos dispositivos móveis (celulares e $p d a$ 's) apresenta um visor reduzido, tem limitação com relação à entrada de dados (script pen ou pequenos botões) e não tem a mesma capacidade de processamento de um computador convencional (modelo desktop). Somente a parte realmente significativa do sistema de informação deve ser levada para o usuário por meio destes dispositivos.

\subsection{Componentes dos sistemas pervasivos}

Basicamente, há dois conceitos importantes para construção de aplicações pervasivas no contexto deste trabalho: componentes de software e sistemas distribuídos. 
Um componente de software é um conjunto de código que encapsula uma ou funcionalidades. Segundo Yacoub [Yacoub 1995], os componentes têm três descriạis básicas: informal, externa e interna. A descrição informal busca responder as quescọes relacionais entre o homem e o componente. A descrição externa define, formalmetões comunicação entre o componente e a plataforma por este utilizado. Finalmente, a características internas refletem aspectos internos do componente. Já Brown [Brown 1999] além de reafirmar as características citadas acima assevera que para assegurar-se de que9] sistema de software, baseado em componente possa funcionar correta e eficazme um descrição externa é o fator mais importante a ser observado, já que esta parte terá o pape, a comunicar-se com a arquitetura do sistema.

Um software desenvolvido para um ambiente pervasivo pode ser construído a partir de pequenos componentes, chamados facets [Belamani 2003]. Várias adaptações precisam sertir realizadas para que estes se comuniquem com uma aplicação back office. Tais adaptações incluem: adaptação de dados, adaptação do nível de rede, adaptação de energia (entende-se por energia o consumo da bateria de um dispositivo portátil/móvel), adaptação de migração e finalmente, adaptação de funcionalidade [Belamani 2003].

Os sistemas pervasivos estão fortemente relacionados aos sistemas distribuídos, pois os equipamentos portáteis não disponibilizam um volume de memória e de processamento adequados, restando então fazer chamada remota, ou seja, invocar um procedimento que é executado em um computador e retornar somente os dados para o dispositivo móvel/portátil.

Existem diversas tecnologias para construção de aplicações distribuídas, tais como RMI [Grosso 2001], CORBA [Orfalli, 1998] entre outras.

\section{Desenvolvimento distribuído de componentes pervasivos}

\subsection{Processo distribuído de software}

O desenvolvimento distribuído ocorre quando vários nós concorrem para desenvolver um mesmo produto ou parte dele. Neste cenário, a complexidade do processo de desenvolvimento se amplia. A redução do tempo é a principal razão da divisão de tarefas na produção distribuida de software, porém o tempo de comunicação e de resposta entre os nós podem ser incomensuráveis. Há muitas variáveis neste cenário, tais como a cultura, a língua, a capacidade de cada nó entre outras. [Herbsleb 2003][Suzuki, 1999]. Além disso, desenvolver software em uma rede de produção requer gerenciamento mais eficaz de tarefas [Jäger 2001], ferramentas, capacidades e informação. [Martin 1996]

Embora a internet ofereça inúmeras vantagens em relação à comunicação, um projeto de software é muito complexo e grande, surgindo então uma necessidade de framework [Suzuki, 1999]. Este deve esclarecer como a informação é gerenciada na rede e como as tarefas são delegadas, por exemplo.

Para o desenvolvimento desta aplicação (ManWApp), foram escolhidas duas variáveis críticas de controle de processo que compõem um framework. Tais variáveis são gestão de tarefas e gestão de repositório. Todas as variáveis críticas eram compartilhadas e todos os nós tinham a mesma hierarquia dentro da rede de produção.

Nesta rede a gestão de tarefas teve o objetivo de distribuir e rearranjar atividades de desenvolvimento por todos os nós. A delegação de tarefas foi baseada na capacidade de desenvolvimento individual e no tempo de entrega do subproduto de cada nó. Algumas inldades foram encontradas na dependência de atividades. Por módulo web service fosse dificuldades dianalida de um dispositivo móvel era preciso que um modulo we

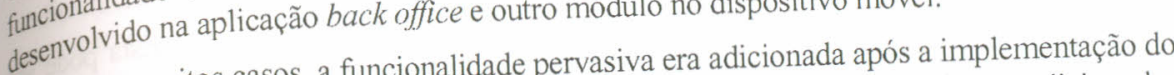

Em muitos casos, a funcionalidade pervasiva era adicionada aós a implem dicionadas Enm modelo clássico (desktop). Por isso, algumas funcionalidades foram adicionadas sistema no mo ao projeto back office para suportar informações. Por exemplo, em alguns dispositivos móveis a os foi necessário compatibilizar informaça decimais, enquanto que no casos foi possível tratar números com grande $\mathrm{q}$

naão for poste tipo de tratamento é convencional.

A A heterogeneidade dos nos da rede de producto final. resolver os impasses tende a sera, ou seja, tempo médio que um nó leva para responder a um

A latência de resposta, ou sja, temo a utilização de estimulo da rede é difícil de ser calculado. Uma solução encontra uma nova versão no checkpoints : um nó gera um subproduto toda vez que armazena unamento de versões e repositório. A escolha de um repositório centralizado facilitou o gerenciamen nó. O repositório evitou ambiguidades, provendo ras do projeto, identificando qual nó estava alterando qual gerenciou arquivo.

Assim que um ciclo de atividades, de acordo com o projeto, fosse concluído, uma testes com usuários e demais membros do grupo. vers servidor de aplicações. Este processo está ilustrado na Figura 2

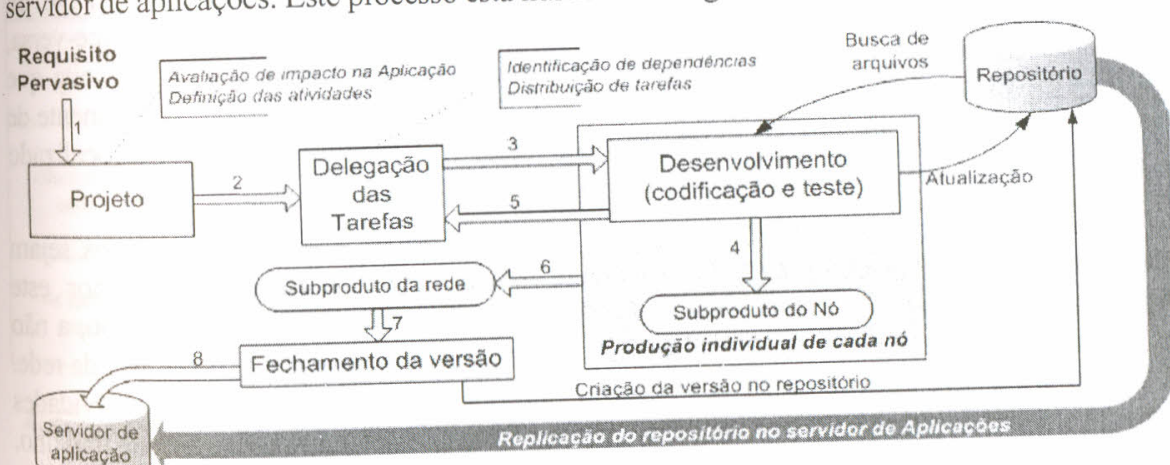

Figura 2 - Processo adotado para o estudo de caso

fluxo 1 da Figura 2 indica a introdução de um requisito pervasivo, ou seja, a necessida de um dispositivo móvel/portátil no projeto. Após determinar as especificações pervasivas, fase de projeto ilustrada pela Figura 2. um conjunto de atividades, fluxo 2 da Figura 2 é então selecionado para distribuição.

A diviño como norteador o diagrama de seqüênci. Este pode representar a interação entre os módulos funcionais e camadas do sistem. Sendo assim, a delegação de tarefas foi condicionada a capacidade que cada nó tem sistema. Sendo assim, a delegação de tarefas foi condicionada a capacide gubproduto do no. em produzir determinada funcionalidade e o tempo estimado de entrega do sut Uma análise de impacto de acordo com a agregação da funcionalidade, discutido a seguir no item 3 . Cada nó, recebia como tarefa, a codificação de um , era feita em um componente pervasian o mesmo objeto era desenvolvido 

por vários nós, no entanto, um grau mais refinado deste mesmo diagrama de sequiência
elaborado exclusivamente para tal objeto.

O fluxo 3 da Figura 2, identifica a tarefa sendo enviada para um nó. Esta pode cumprida ou não, dependendo da capacidade e do tempo de desenvolvimento. Code ser desenvolvido, o nó gera um subproduto, fluxo 4 da Figura 2, e atualiza um repositório seja de projeto. Caso o nó não cumpra o desenvolvimento, a tarefa é novamente delegro central da Figura 2 Quando um nó termina por completo fluxo 6 da Figura 2. Quando todos os nós concluírem suas tonproduto, versão, fluxo 7 da Figura 2. O repositório é notificado para manter estarre o fechamento da então disponibilizada no servidor de aplicaçoes para uso, fluxo 8 da Figura 2 . e a aplicação é

\subsection{Agregação de funcionalidades a dispositivos móveis}

Os componentes pervasivos desenvolvidos neste estudo de caso foram avaliados principalmente sob dois pontos de vista: interação com o equipamento e interação com sistema back office. Além disso, foram identificadas quatro maneiras de como os componentes podem agregar funcionalidades. São elas: agregacão por componente os agregação por sincronização on-line, agregação por sincronização off-line e agregação por chamada remota.

Na agregação local o componente de software interage somente com o dispositivo móvel/portátil. Na agregação por sincronização as informações são trocadas entre aplicativo back office e aplicativo pervasivo, resultando em atualizações no banco de dados da aplicação back office e/ou da aplicação pervasiva. A sincronização on-line ocorre automaticamente, ou seja, os dados que estão no dispositivo são transferidos para outro sistema ou vice-versa, assim que uma comunicação estiver disponível. No modelo off-line, o usuário solicita seja feita uma sincronização. A agregação por chamada remota implica que o componente de software instalado no dispositivo móvel invoque remotamente um procedimento localizado no back office.

O componente que utiliza agregação local deve prever que todos os dados sejam armazenados no dispositivo e todo o processamento seja efetuado também por este dispositivo. Neste caso, o componente não sofre interação com outro sistema externo, a não ser pelo usuário. Um mesmo componente pode ser desenvolvido por um ou mais nós da rede. Quando vários nós desenvolvem um mesmo componente, a dependência de atividades aumenta e consequentemente, o repositório é fundamental para evitar duplicações de trabalho. O projeto todo fica centrado no dispositivo móvel. Todos os requisitos não funcionais estão centrados nas limitações do equipamento.

Com exceção da agregação local, todos os demais tipos de agregação necessitam de que a aplicação back office e a aplicação instalada no dispositivo móvel/portátil estejam preparadas para troca de dados. O diferencial entre um tipo e outro de agregação é a disponibilidade do serviço de sincronização e a capacidade de processamento/armazenamento de informações do dispositivo móvel

$\mathrm{Na}$ agregação por sincronização off-line, o dispositivo portátil deve comportar 0 processamento e não necessariamente deve armazenar todas as informacões, mas somente as necessárias. Em muitos casos, as informações são resultados de consultas a várias tabelas, parte de uma tabela no banco de dados, e outras consultas. indiretamente com outra aplicação. O serviço de sincronização não necesariamente precis atar disponível o tempo todo. Os dados armazenados no dispositivo pervasivo devem ser estar dispomente completos para o usuário trabalhar, porém precisam ser pequenos o bastante suficiene dispositivo móvel suporte-os.

$\mathrm{Na}$ sincronização on-line, o dispositivo deve comportar o processamento do

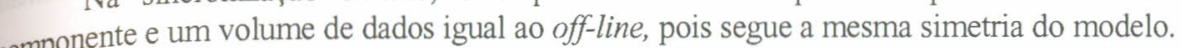
compon em alguns casos, o volume de dados que o dispositivo precisa armazenar pode ser porem, enis, toda vez que uma informação não estiver disponível no banco de dados do dispositivo móvel, uma conexão de rede é estabelecida e o banco de dados local é atualizado. Esta aplicação é decorrente da interação com o back office.

$\mathrm{Na}$ agregação por chamada remota, o facet invoca remotamente um procedimento. $\mathrm{O}$ dispositivo deve ter capacidade suficiente para processar o volume de dados gerados pelo procedimento remoto. Diretamente o componente interage com outra aplicação e, além disso, o sistema back office deve disponibilizar sempre o serviço de troca de dados.

A tabela 2 mostra os modelos de agregação, sua avaliação com relação à interação com o dispositivo e ao sistema back office e as tarefas a serem cumpridas. Um facet pode ser construído também utilizando mais de um modelo de agregação.

Tabela 1 - Modelos de agregação de funcionalidade em dispositivos móveis

\begin{tabular}{|c|c|c|c|}
\hline $\begin{array}{l}\text { Modelo de } \\
\text { agregação }\end{array}$ & Interação com o dispositivo & $\begin{array}{c}\text { Interação com o } \\
\text { sistema back office }\end{array}$ & Tarefas \\
\hline 1. Local & $\begin{array}{l}\text { Amazenamento de todia a } \\
\text { informação } \\
\text { Processamento de todas as } \\
\text { funcionalidades }\end{array}$ & - & - Desenvolver um facet: acesso a recursos locais \\
\hline $\begin{array}{l}\text { 2-Sincronização } \\
\text { off-line }\end{array}$ & $\begin{array}{l}\text { Armazenamento parcial de } \\
\text { informaç̄és } \\
\text { Processamento de } \\
\text { funcionalidades locais } \\
\end{array}$ & $\begin{array}{l}\text { Disponibilidade } \\
\text { esporádica de } \\
\text { sincronização }\end{array}$ & $\begin{array}{l}\text { - Desenvolver um facet: acesso ao banco de dados local } \\
\text { - Desenvolver o componente de sincronização no sistema } \\
\text { back office }\end{array}$ \\
\hline $\begin{array}{l}\text { 3-Sincronizaçâo } \\
\text { on-line }\end{array}$ & $\begin{array}{l}\text { Armazenamento parcial de } \\
\text { informaç̄ess } \\
\text { Processamento de } \\
\text { funcionalidades locais }\end{array}$ & $\begin{array}{l}\text { Média disponibilidade } \\
\text { frequente de } \\
\text { sincronização }\end{array}$ & $\begin{array}{l}\text { - Desenvolver um facet: acesso ao banco de dados local; } \\
\text { solicitação automática de sincronizaçâo quando os dados } \\
\text { estão desatualizados ou incompletos. } \\
\text { - Desenvolver o componente de sincronização no sistema } \\
\text { back office }\end{array}$ \\
\hline $\begin{array}{l}\text { 4Chamada } \\
\text { remota }\end{array}$ & $\begin{array}{l}\text { Nenhum armazenamento } \\
\text { Processamento de informaç̃es } \\
\text { resultantes da chamada remota }\end{array}$ & $\begin{array}{l}\text { Alta disponibilidade } \\
\text { freqüente de troca de } \\
\text { dados }\end{array}$ & $\begin{array}{l}\text { - Desenvolver um facet: capacidade de processar um } \\
\text { volume razóável de informaç̃és } \\
\text { - Desenvolver o componente capaz de disponibilizar um } \\
\text { web service }\end{array}$ \\
\hline
\end{tabular}

\section{Conclusões}

Este artigo apresentou o estudo de caso do desenvolvimento distribuído de uma aplicação pervasiva chamada ManWApp. O desenvolvimento em rede de produção pode reduzir consideravelmente o tempo de entrega do produto final, desde que um framework seja detalhado antes do desenvolvimento de software. Neste estudo de caso os pesquisadores e desenvolvedores residem em locais distantes (acima de $400 \mathrm{~km}$ ), o que obrigou a adoção de procedimentos para desenvolver software em uma rede de produção. Um processo precisou ser desenvolvido (Figura 2) para solucionar o problema da descentralização das pessoas envolvidas.

Foram impostas algumas modificações no projeto, tais como novos requisitos pervasivos e novos requisitos funcionais. Neste caso, após reavaliação do projeto, foi percebido que a dependência de atividades era muito grande, causando desenvolvimento seqüencial em algumas etapas. Se a alteração de uma regra de negócio causasse algum impacto em um facet, o desenvolvimento seria praticamente seqüencial. 
A grande vantagem neste processo está na sua simplicidade e agilidade. Em curtos espaços de tempo, os nós já tinham subprodutos que eram armazenados e disponibilizados em um repositório. No entanto, periodicamente era necessário uma reunião com os projetistas, pois o processo descrito neste trabalho aborda o desenvolvimento, não tratando as demais áreas do projeto.

Do ponto de vista do usuário final, a vantagem do desenvolvimento descentralizadoé que o tempo de entrega de uma nova funcionalidade pode ser reduzido drasticamente. Uma rede de produção de software pode ser constituída de vários nós com vários especialistas diferentes. Além disso, a tarefa pode ser delegada de acordo com a capacidade, o tempo de entrega e o fuso horário onde se encontra o nó.

O processo apresentado neste artigo aborda somente o desenvolvimento distribuido. A próxima etapa é desenvolver um processo, compativel a este, porém capaz de atingir uma escala de análise e projeto.

\section{Referencias}

Belamani, et al. (2003) "Dynamic component composition for functionality adaptation in pervasive environments". IEEE Workshop of future trends of Distributed Computing Systems.

Brown, A. K. Wallnau. (1999) "The Current State of CBSE". IEEE Software, 15 (5): 37-46, Sep-Oct.

Burkhardt, Jochen , et al. (2002) "Pervasive computing - technology and architecture of mobile internet applications". Addison Wesley.

Grosso, Willian. (2001) "Java RMI. Oreilly \& Assoc." 1' edição.

Hansmann, Uwe et al. (2001) "Pervasive computing - the mobile world". Springer 2a . Edição

Herbsleb James D. Morcrkus, Audris. (2003) "An empirical Study of Speed and Communication in Globally Distributed Software Development". IEEE transactions on Software Engineering, June n.6 vol29 pp. 481-494

Jäger, et al. (2001) "A delegation based model for distributed software process management." EWSPT Springer-Verlag Berlin.

Martin, Patric e Poweley, Wendy. (1996) "A management information repository form distribute application management". in ACM Conference of the Centre for Advanced Studies on Collaborative research

Newcomer, Eric. (2002) "Understanding Web Services," $1^{a}$ edição. David Chappel Series Editor.

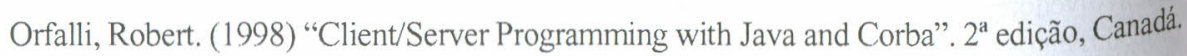

Suzuki, Junichi e Uamamoto Yoshikazy. (1999) "Leveraging Distributed Software Development". IEEE computer, September. Vol32 pp. 59 - 65

Yacoub, Sherif. Ammar, Hany e Mili, Ali. (1995) "Characterizing a Software Component". http://www.sei.cmu.edu/cbs/icse99/papers/34/34.htm, June. 